\title{
Simultaneous Description of Even-Even, Odd-Mass and Odd-Odd Nuclear Spectra
}

\author{
H. G. Ganev and A. I. Georgieva \\ Institute of Nuclear Research and Nuclear Energy, Bulgarian Academy of Sciences, Sofia 1784, Bulgaria
}

\begin{abstract}
The orthosymplectic extension of the Interacting Vector Boson Model (IVBM) is used for the simultaneous description of the spectra of different families of neighboring heavy nuclei. The structure of even-even nuclei is used as a core on which the collective excitations of the neighboring odd-mass and odd-odd nuclei are built on. Hence, the spectra of the odd-mass and odd-odd nuclei arise as a result of the consequent and self-consistent coupling of the fermion degrees of freedom of the odd particles, specified by the fermion sector $S^{F}(2 \Omega) \subset \operatorname{OSp}(2 \Omega / 12, R)$, to the boson core which states belong to an $S p^{B}(12, R)$ irreducible representation.

The theoretical predictions for different low-lying collective bands with positive and negative parity for two sets of neighboring nuclei with distinct collective properties are compared with experiment and IBM/IBFM/IBFFM predictions. The obtained results reveal the applicability of the used dynamical symmetry of the model.
\end{abstract}

Keywords: Dynamical symmetries, Algebraic models, Collective states, Nuclear Structure

PACS: 21.10.Re,23.20.Lv,21.60.Fw,27.70.+q,27.60.+j

\section{INTRODUCTION}

In recent years, extensive experimental evidence for the existence of distinct band structures in different even-even, odd-mass and odd-odd nuclei has been obtained. It has created an opportunity for testing the predictions of different theoretical models on the level properties of these nuclei. Generally, it is believed that the properties of the odd-mass nuclei can be described using particle-core coupled-type models. In this respect, it was found in nuclear physics that the combined algebras of bosons and fermions are particular useful in a description of the spectra of the odd-mass nuclei. Moreover, an attempt to get a classification of several nuclei including even and odd ones have been realized and very interesting results have been obtained [1], [2]. The main attention has been paid to the investigations of the models with the Hamiltonians, which exhibit dynamical supersymmetries. Such Hamiltonians are presented by the sums of the Casimir operators for an appropriated subgroup chain of a certain supergroup. The spectra of even-even and odd-mass nuclei, which states are unified into a common supermultiplet, are described by the same Hamiltonian with a single set of adjustable parameters.

In the early 1980s, a boson-number-preserving version of the phenomenological algebraic Interacting Vector Boson Model (IVBM) [3] was introduced and applied successfully [4] to a description of the low-lying collective rotational spectra of the even-even medium and heavy mass nuclei. With the aim of extending these applications to incorporate new experimental data on states with higher spins and to incorporate new excited bands, we explored the symplectic extension of the IVBM [5], for which the dynamical symmetry group is $\operatorname{Sp}^{B}(12, R)$. This extension is realized from, and has its physical interpretation over basis states of its maximal compact subgroup $U^{B}(6) \subset S p^{B}(12, R)$, and resulted in the description of various excited bands of both positive and negative parity of complex systems exhibiting rotationvibrational spectra [6]. In [7] an orthosymplectic extension of the IVBM was carried out in order to encompass the treatment of the odd-mass nuclei. In [8] the investigation of the doublet bands in doubly odd nuclei from $A \sim 130$ region was presented. A good agreement between experiment and theoretical predictions for the energy levels of these bands as well as in-band $B(E 2)$ and $B(M 1)$ transition probabilities is obtained. With the present work we exploit further the boson-fermion extension [7] of IVBM for the simultaneous description of the low-lying positive and/or negative bands of two different sets of neighboring even-even, odd-mass and odd-odd nuclei with various collective properties. The first set of nuclei which we choose possesses a clear rotational character, whereas the second one displays typical vibrational features. The theoretical description of the doubly odd nuclei under consideration is fully consistent and starts with the calculation of theirs even-even and odd-mass neighbors. We consider the simplest physical picture in which two particles (or quasiparticles) with intrinsic spins taking a single $j$-value are coupled to an even-even core nucleus which states belong to an $S p^{B}(12, R)$ irreducible representation (irrep). Thus, the bands of the odd-mass and 
odd-odd nuclei arise as collective bands built on a given even-even nucleus. Nevertheless, the results for the energy spectra obtained in this simplified version of the model agree rather well with the experimental data.

\section{THE EVEN-EVEN CORE NUCLEI}

The algebraic structure of the IVBM is realized in terms of creation and annihilation operators of two kinds of vector bosons $u_{m}^{+}(\alpha), u_{m}(\alpha)(m=0, \pm 1)$, which differ in an additional quantum number $\alpha= \pm 1 / 2$-the projection of the $T$-spin (an analogue to the $F$-spin of IBM-2). The bilinear products of the creation and annihilation operators of the two vector bosons generate the boson representations of the non-compact symplectic group $S p^{B}(12, R)$ [3]:

$$
\begin{aligned}
F_{M}^{L}(\alpha, \beta) & =\sum_{k, m} C_{1 k 1 m}^{L M} u_{k}^{+}(\alpha) u_{m}^{+}(\beta), \\
G_{M}^{L}(\alpha, \beta) & =\sum_{k, m} C_{1 k 1 m}^{L M} u_{k}(\alpha) u_{m}(\beta), \\
A_{M}^{L}(\alpha, \beta) & =\sum_{k, m} C_{1 k 1 m}^{L M} u_{k}^{+}(\alpha) u_{m}(\beta),
\end{aligned}
$$

where $C_{1 k 1 m}^{L M}$, which are the usual Clebsch-Gordon coefficients for $L=0,1,2$ and $M=-L,-L+1, \ldots L$, define the transformation properties of (1) and (2) under rotations. Being a noncompact group, the unitary representations of $S p^{B}(12, R)$ are of infinite dimension, which makes it impossible to diagonalize the most general Hamiltonian. When restricted to the group $U^{B}(6)$, each irrep of the group $S p^{B}(12, R)$ decomposes into irreps of the subgroup characterized by the partitions [5],[9]: $\left[N, 0^{5}\right]_{6} \equiv[N]_{6}$, where $N=0,2,4, \ldots$ (even irrep) or $N=1,3,5, \ldots$ (odd irrep). The subspaces $[N]_{6}$ are finite dimensional, which simplifies the problem of diagonalization. Therefore the complete spectrum of the system can be calculated through the diagonalization of the Hamiltonian in the subspaces of all the unitary irreducible representations (UIR) of $U^{B}(6)$, belonging to a given UIR of $S p^{B}(12, R)$, which further clarifies its role of a group of dynamical symmetry.

The most important application of the $U^{B}(6) \subset S p^{B}(12, R)$ limit of the theory is the possibility it affords for describing both even and odd parity bands up to very high angular momentum [5]. In order to do this we first have to identify the experimentally observed bands with the sequences of basis states of the even $S p^{B}(12, R)$ irrep (Table 1). As we deal with the symplectic extension we are able to consider all even eigenvalues of the number of vector bosons $N$ with the corresponding set of $T$-spins, which uniquely define the $S U^{B}(3)$ irreps $(\lambda, \mu)$. The multiplicity index $K$ appearing in the final reduction to the $S O^{B}(3)$ is related to the projection of $L$ on the body fixed frame and is used with the parity $(\pi)$ to label the different bands $\left(K^{\pi}\right)$ in the energy spectra of the nuclei. For the even-even nuclei we have defined the parity of the states as $\pi_{\text {core }}=(-1)^{T}$ [5]. This allowed us to describe both positive and negative bands.

Further, we use the algebraic concept of "yrast" states, introduced in [5]. According to this concept we consider as yrast states the states with given $L$, which minimize the energy with respect to the number of vector bosons $N$ that build them. Thus the states of the ground state band (GSB) were identified with the $S U^{B}(3)$ multiplets $(0, \mu)[5]$. In terms of $(N, T)$ this choice corresponds to $(N=2 \mu, T=0)$ and the sequence of states with different numbers of bosons $N=0,4,8, \ldots$ and $T=0$ (and also $T_{0}=0$ ). The presented mapping of the experimental states onto the $S U^{B}(3)$ basis states, using the algebraic notion of yrast states, is a particular case of the so called "stretched" states [10]. The latter are defined as the states with $\left(\lambda_{0}+2 k, \mu_{0}\right)$ or $\left(\lambda_{0}, \mu_{0}+k\right)$, where $N_{i}=\lambda_{0}+2 \mu_{0}$ and $k=0,1,2,3, \ldots$. In the symplectic extension of the IVBM the change of the number $k$, which is related in the applications to the angular momentum $L$ of the states, gives rise to the collective bands.

It was established [11] that the correct placement of the bands in the spectrum strongly depends on their bandheads' configuration, and in particular, on the minimal or initial number of bosons, $N=N_{i}$, from which they are built. The latter determines the starting position of each excited band. In the present application we take for $N_{i}$ the value at which the best $\chi^{2}$ is obtained in the fitting procedure for the energies of the considered excited band.

To describe the structure of odd-mass and odd-odd nuclei, first a description of the appropriate even-even cores should be obtained. We use the unitary limit of IVBM [5] and determine the values of five phenomenological model parameters, related to the boson degrees of freedom (see Eq. (11)), by fitting the energies of the ground and few excited bands ( $\gamma-$ and/or $\beta$ - bands) of the even-even nuclei to the experimental data [12], using a $\chi^{2}$ procedure. The theoretical predictions for the even-even core nuclei are presented in Figs. 1(a) and 1(b). For comparison, the predictions of IBM are also shown. From the figures one can see that the calculated energy levels agree rather well up to very high angular momenta with the observed data. One can see also that for high spins $(L \geq 10-14)$, where the deviations of the IBM predictions become more significant, the structure of the energy levels of the GSB ( $\beta-$ and $\gamma$-bands) is reproduced rather well. The ${ }^{156} G d$ core nucleus shows a well-developed ground state rotational band, 
whereas the ${ }^{114} S n$ one shows a purely vibrational (equidistant) spectrum as can be clearly seen from Fig. 1(b). The latter is described in IBM in its $U(5)$ dynamical symmetry limit which is reasonable for spherical nuclei in the $S n-S b$ region.
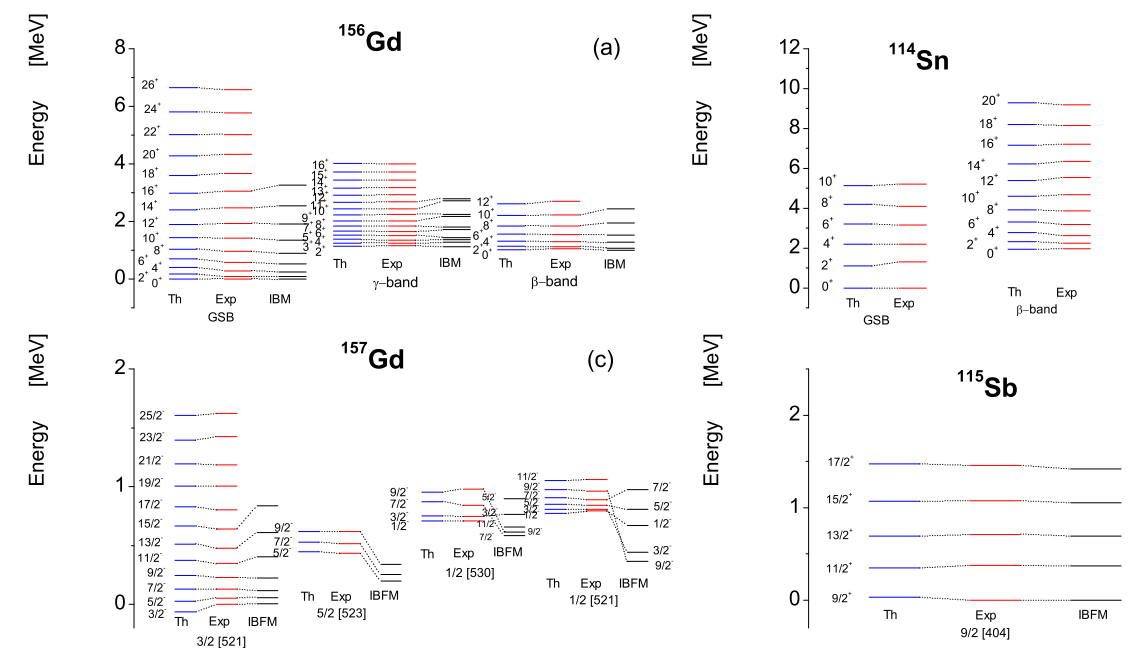

(b)
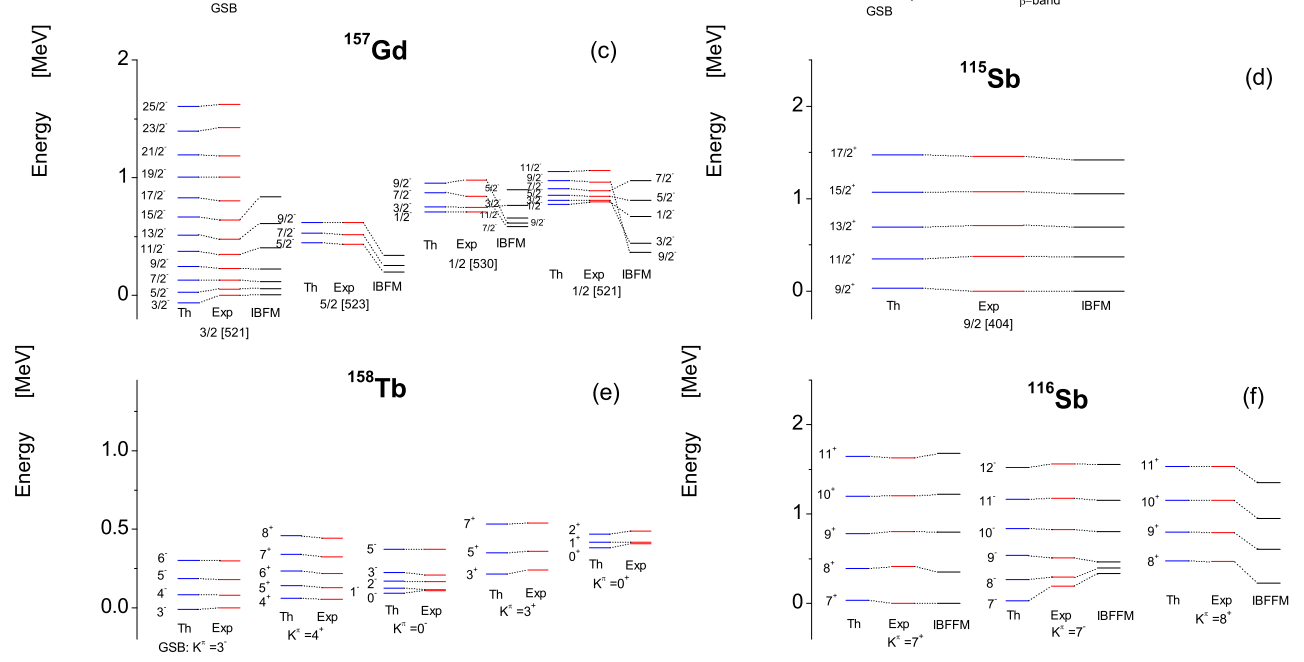

FIGURE 1. Comparison of the theoretical, experimental and IBM/IBFM/IBFFM energies for the ground and first excited positive and/or negative parity bands of ${ }^{156} \mathrm{Gd}(\mathrm{a}),{ }^{157} \mathrm{Gd}(\mathrm{c}),{ }^{158} \mathrm{~Tb}(\mathrm{e})$, ground and first excited $\beta$ bands of ${ }^{114} \mathrm{Sn}(\mathrm{b}), K_{J}^{\pi}=9 / 2^{+}$intruder band of ${ }^{115} \mathrm{Sb}(\mathrm{d})$, and the intruder $K_{J}^{\pi}=7^{+}, K_{J}^{\pi}=7^{-}$and $K_{J}^{\pi}=8^{+}$bands of ${ }^{116} \mathrm{Sb}$ (f).

\section{ORTHOSYMPLECTIC EXTENSION}

In order to incorporate the intrinsic spin degrees of freedom into the symplectic IVBM, we extend the dynamical algebra of $S^{B}(12, R)$ to the orthosymplectic algebra of $\operatorname{SSp}(2 \Omega / 12, R)$ [7]. For this purpose we introduce a particle (quasiparticle) with spin $j$ and consider a simple core plus particle picture. Thus, in addition to the boson collective degrees of freedom we introduce creation and annihilation operators $a_{m}^{\dagger}$ and $a_{m}(m=-j, \ldots, j)$, which satisfy the anticommutation relations: $\left\{a_{m}, a_{m^{\prime}}^{\dagger}\right\}=\delta_{m m^{\prime}}$ and $\left\{a_{m}^{\dagger}, a_{m^{\prime}}^{\dagger}\right\}=\left\{a_{m}, a_{m^{\prime}}\right\}=0$.

All bilinear combinations of $a_{m}^{+}$and $a_{m^{\prime}}$, namely

$$
\begin{aligned}
& f_{m m^{\prime}}=a_{m}^{\dagger} a_{m^{\prime}}^{\dagger}, \quad m \neq m^{\prime} \\
& g_{m m^{\prime}}=a_{m} a_{m^{\prime}}, \quad m \neq m^{\prime} ; \\
& C_{m m^{\prime}}=\left(a_{m}^{\dagger} a_{m^{\prime}}-a_{m^{\prime}} a_{m}^{\dagger}\right) / 2
\end{aligned}
$$

generate the (Lie) fermion pair algebra of $S O^{F}(2 \Omega)$. Their commutation relations are given in [7]. The number preserving operators (4) generate maximal compact subalgebra of $S O^{F}(2 \Omega)$, i.e. $U^{F}(\Omega)$. 


\section{Fermion dynamical symmetries}

One can further construct a certain fermion dynamical symmetry, i.e. the group-subgroup chain:

$$
S O^{F}(2 \Omega) \supset G^{\prime} \supset G^{\prime \prime} \supset \ldots
$$

In particular for one particle occupying a single level $j$ we are interested in the following dynamical symmetry:

$$
S O^{F}(2 \Omega) \supset S p(2 j+1) \supset S U^{F}(2),
$$

where $\operatorname{Sp}(2 j+1)$ is the compact symplectic group. The dynamical symmetry (6) remains valid also for the case of two particles occupying the same level $j$. In this case, the allowed values of the quantum number $I$ of $S U^{F}(2)$ in (6) according to reduction rules are $I=0,2, \ldots, 2 j-1$ [13]. If the two particles occupy different levels $j_{1}$ and $j_{2}$ of the same or different major shell(s), one can consider the chain

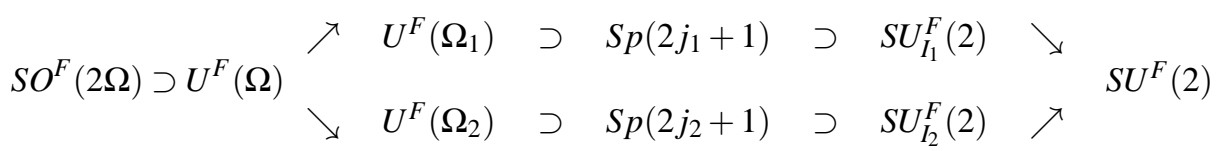

where $\Omega=\Omega_{1}+\Omega_{2}$. For simplicity hereafter we will use just the reduction $S O^{F}(2 \Omega) \supset S U^{F}(2)$ (i.e. dropping all intermediate subgroups between $S O^{F}(2 \Omega)$ and $S U^{F}(2)$ ) and keep in mind the proper content of the set of $I$ values for one and/or two particles cases, respectively.

\section{Dynamical Bose-Fermi symmetry. Dynamical supersymmetry}

Once the fermion dynamical symmetry is determined we proceed with the construction of the Bose-Fermi symmetries. If a fermion is coupled to a boson system having itself a dynamical symmetry (e.g., such as an IBM core), the full symmetry of the combined system is $G^{B} \otimes G^{F}$. Bose-Fermi symmetries occur if at some point the same group appears in both chains $G^{B} \otimes G^{F} \supset G^{B F}$, i.e. the two subgroup chains merge into one.

The standard approach to supersymmetry in nuclei (dynamical supersymmetry) is to embed the Bose-Fermi subgroup chain of $G^{B} \otimes G^{F}$ into a larger supergroup G, i.e. $G \supset G^{B} \otimes G^{F}$. Making use of the embedding $S U^{F}(2) \subset$ $S O^{F}(2 \Omega)$ we make orthosymplectic (supersymmetric) extension of the IVBM which is defined through the chain [7]:

$$
\begin{array}{cccc}
\operatorname{OSp}(2 \Omega / 12, R) \quad \supset \quad & S^{F}(2 \Omega) & \otimes & S^{B}(12, R) \\
& \Downarrow & \\
& & & U^{B}(6) \\
& & N \\
& & \Downarrow \\
& S U^{F}(2) & \otimes & S U^{B}(3) \otimes U_{T}^{B}(2) \\
& I & & (\lambda, \mu) \Longleftrightarrow(N, T) \\
& \searrow & & \Downarrow \\
& & & S^{B}(3) \otimes U^{B}(1) \\
& & L & T_{0} \\
& \Downarrow & & \\
& \operatorname{Spin}^{B F}(3) & \supset & \operatorname{Spin}^{B F}(2), \\
J & & J_{0}
\end{array}
$$

where below the different subgroups the quantum numbers characterizing their irreducible representations are given. $\operatorname{Spin}^{B F}(n)(n=2,3)$ denotes the universal covering group of $S O(n)$.

\section{THE ENERGY SPECTRA OF ODD-MASS AND ODD-ODD NUCLEI}

We can label the basis states according to the chain (8) as:

$$
\left|[N]_{6} ;(\lambda, \mu) ; K L ; I ; J J_{0} ; T_{0}\right\rangle \equiv\left|[N]_{6} ;(N, T) ; K L ; I ; J J_{0} ; T_{0}\right\rangle,
$$


where $[N]_{6}$-the $U^{B}(6)$ labeling quantum number and $(\lambda, \mu)$-the $S U^{B}(3)$ quantum numbers characterize the core excitations, $K$ is the multiplicity index in the reduction $S U^{B}(3) \supset S O^{B}(3), L$ is the core angular momentum, $I-$ the intrinsic spin of an odd particle (or the common spin of two fermion particles for the case of odd-odd nuclei), $J, J_{0}$ are the total (coupled boson-fermion) angular momentum and its third projection, and $T, T_{0}$ are the $T$-spin and its third projection, respectively.

The Hamiltonian of the combined boson-fermion system can be written as linear combination of the Casimir operators of the different subgroups in (8):

$$
H=a N+b N^{2}+\alpha_{3} T^{2}+\beta_{3}^{\prime} L^{2}+\alpha_{1} T_{0}^{2}+\eta I^{2}+\gamma^{\prime} J^{2}+\zeta J_{0}^{2}
$$

and it is obviously diagonal in the basis (9) labeled by the quantum numbers of their representations. Then the eigenvalues of the Hamiltonian (10), that yield the spectrum of the odd-mass and odd-odd systems are:

$$
E\left(N ; T, T_{0} ; L, I ; J, J_{0}\right)=a N+b N^{2}+\alpha_{3} T(T+1)+\beta_{3}^{\prime} L(L+1)+\alpha_{1} T_{0}^{2}+\eta I(I+1)+\gamma^{\prime} J(J+1)+\zeta J_{0}^{2} .
$$

The infinite set of basis states classified according to the reduction chain (8) are schematically shown in Table 1.

TABLE 1. Classification scheme of basis states (9) according the decompositions given by the chain (8).

\begin{tabular}{l|l|l|l|l|l}
\hline$N$ & $T$ & $(\lambda, \mu)$ & $K$ & $L$ & $J=L \pm I$ \\
\hline \hline \multirow{2}{*}{0} & 0 & $(0,0)$ & 0 & 0 & $I$ \\
\hline \multirow{2}{*}{2} & 1 & $(2,0)$ & 0 & 0,2 & $I ; 2 \pm I$ \\
\cline { 2 - 6 } & 0 & $(0,1)$ & 0 & 1 & $1 \pm I$ \\
\hline \multirow{5}{*}{4} & 2 & $(4,0)$ & 0 & $0,2,4$ & $I ; 2 \pm I ; 4 \pm I$ \\
\cline { 2 - 6 } & 1 & $(2,1)$ & 1 & $1,2,3$ & $1 \pm I ; 2 \pm I ; 3 \pm I$ \\
\cline { 2 - 6 } & 0 & $(0,2)$ & 0 & 0,2 & $I ; 2 \pm I$ \\
\hline \multirow{2}{*}{$:$} & $\vdots$ & $\vdots$ & $\vdots$ & $\vdots$ & $\vdots$ \\
\hline
\end{tabular}

The basis states (9) can be considered as a result of the coupling of the orbital $\left|(N, T) ; K L M ; T_{0}\right\rangle$ and spin $\phi_{j \equiv I, m}$ wave functions. Then, if the parity of the single particle is $\pi_{s p}$, the parity of the collective states of the odd $-A$ nuclei will be $\pi=\pi_{c o r e} \pi_{s p}$ [7]. In analogy, one can write $\pi=\pi_{c o r e} \pi_{s p}(1) \pi_{s p}(2)$ for the case of odd-odd nuclei. Thus, the description of the positive and/or negative parity bands requires only the proper choice of the core band heads, on which the corresponding single particle(s) is (are) coupled to, generating in this way the different odd- $A$ (odd-odd) collective bands.

In our application, the most important point is the identification of the experimentally observed states with a certain subset of basis states from (ortho)symplectic extension of the model. In general, except for the excited bands of the even-even nuclei for which the stretched states of the first type ( $\lambda$-changing) are used, the stretched states of the second type ( $\mu$-changing) are considered in all the calculations of the collective states of the odd-mass and doubly odd nuclei.

Additionally to the five parameters $a, b, \alpha_{3}, \beta_{3}, \alpha_{1}$ entering in Eq. (11) which are used to describe the energies of the even-even core nuclei, the number of adjustable parameters needed for the complete description of the collective spectra of both odd-A and odd-odd nuclei is three, namely $\gamma, \zeta$ and $\eta$. The first two are evaluated by a fit to the experimental data [12] of the GSB of the corresponding odd-A neighbor, while the last one is introduced in the final step of the fitting procedure for the odd-odd nucleus, respectively.

The odd-A nucleus ${ }^{157} \mathrm{Gd}$ can be considered as a neutron particle coupled to the ${ }^{156} \mathrm{Gd}$ core, while the ${ }^{115} \mathrm{Sb}$ is obtained by proton coupling to the ${ }^{116} \mathrm{Sn}$, respectively. The comparison between the experimental spectra for the lowlying bands and our calculations for ${ }^{157} G d$ and ${ }^{115} \mathrm{Sb}$ nuclei is illustrated in Figs. 1(c) and 1(d). In our considerations we take into account only the first available single particle orbit $j_{1}$. One can see from the figures that the calculated energy levels agree rather well in general with the experimental data up to very high angular momenta. For comparison, in the figures the IBFM results are also shown. Note that all calculated levels, for the bands considered, are in correct order in contrast to IBFM results (for ${ }^{157} G d$ ). Another difference between the IVBM and IBFM predictions is that in the former the correct placement of all the band heads is reproduced quite well. 
For the calculation of the odd-odd nuclear spectra a second particle should be coupled to the core. In our approach a consistent procedure is employed which includes the analysis of the even-even and odd-mass neighbors of the nucleus under consideration. Thus, as a first step the first odd particle was coupled to the boson core in order to obtain the spectra of the odd-mass neighbors ${ }^{157} G d$ and ${ }^{115} \mathrm{Sb}$. As a second step, we consider the addition of a second particle on a single $j_{2}$ level to the boson-fermion system. The theoretical predictions for the ground and first excited bands of ${ }^{158} \mathrm{~Tb}$ and intruder bands of ${ }^{116} \mathrm{Sb}$ odd-odd nuclei are presented in Figs. 1(e) and 1(f). They are compared with the experimental data and for the ${ }^{116} \mathrm{Sb}$ case also with IBFFM predictions. From the figures one can see the good overall agreement between the theory and experiment which reveals the applicability of the used orthosymplectic extension of the model.

\section{CONCLUSION}

In the present paper, the structure of different collective bands with both positive and negative parity for two sets of neighboring even-even, odd-mass and odd-odd nuclei with various collective properties is presented. The even-even nuclei are used as a core on which the collective excitations of the neighboring odd-mass and odd-odd nuclei are built on. Thus, the spectra of odd-mass and odd-odd nuclei arise as a result of the consequent and self-consistent coupling of the fermion degrees of freedom to the boson core. Hence, a purely collective structure of the bands under consideration is introduced. The good agreement between the theoretical and the experimental band structures confirms the applicability of the used dynamical symmetry of the IVBM. The success is based on the (ortho)symplectic structures of the model which allow the mixing of the basic collective modes - rotational and vibrational ones arising from the yrast conditions - way back on the level of the even-even cores. This allows for the correct reproduction of the high spin states of the collective bands and the correct placement of the different band heads. The simplifications in our approach comes from the fact that only one dynamical symmetry is employed, which leads to exact and simple solutions depending only on the values of the model parameters. The success of the presented application is based also mainly on the proper and consistent mapping of the experimentally observed collective states of the even-even, odd-mass and odd-odd nuclei on the (ortho)symplectic structures. The latter is much simpler approach than the mixing of the basis states considered in other theoretical models.

\section{ACKNOWLEDGMENTS}

This work was supported by the Bulgarian National Foundation for scientific research under Grant Number $\Phi-$ 1501. H. G. G. acknowledges also the support from the European Operational programm HRD through contract $B G 051 P O 001 / 07 / 3.3$ - 02 with the Bulgarian Ministry of Education.

\section{REFERENCES}

1. F. Iachello and A. Arima, The Interacting Boson Model (Cambridge University Press, Cambridge, 1987).

2. F. Iachello and P. Van Isacker, The Interacting Boson Fermion Model (Cambridge University Press, Cambridge, 1991).

3. A. Georgieva, P. Raychev, and R. Roussev, J. Phys. G: Nucl. Part. Phys. 8, 1377 (1982).

4. A. Georgieva, P. Raychev, and R. Roussev, J. Phys. G: Nucl. Part. Phys. 9, 521 (1983).

5. H. Ganev, V. P. Garistov, and A. I. Georgieva, Phys. Rev. C 69, 014305 (2004).

6. A. I. Georgieva, H. G. Ganev, J. P. Draayer and V. P. Garistov, Physics of Particles and Nuclei 40, issue 4, 461 (2009); A. I. Georgieva, H. G. Ganev, J. P. Draayer, and V. P. Garistov, J. Phys.: Conf. Ser. 128, 012032 (2008)

7. H. G. Ganev, J. Phys. G: Nucl. Part. Phys. 35, 125101 (2008).

8. H. G. Ganev, A. I. Georgieva, S. Brant, and A. Ventura, Phys. Rev. C 79, 044322 (2009); H. G. Ganev, A. I. Georgieva, S. Brant, and A. Ventura,Proceedings of 28th International Workshop on Nuclear Theory, Rila Mountains, June 21-27, Bulgaria, (2009).

9. C. Quesne, J. Math. Phys. 14, 366 (1973).

10. D. J. Rowe, Rep. Prog. Phys. 48, 1419 (1985).

11. H. G. Ganev, A. I. Georgieva, and J. P. Draayer, Phys. Rev. C 72, 014314 (2005).

12. Evaluated Nuclear Structure Data File (ENSDF), http://ie.lbl.gov/databases/ensdfserve.html; Level Retrieval Parameters, http://iaeand.iaea.or.at/nudat/levform.html.

13. B. G. Wybourne, Classical Groups for Physicists (Wiley, New York, 1974). 